\title{
Role of time scale in the deformation patterns and hysteresis of NiTi SMA
}

\author{
Qingping SUN ${ }^{1}$ and Yongjun HE
}

Department of Mechanical Engineering, The Hong Kong University of Science and Technology, Hong Kong, China

In this paper we present our recent modelling and experiment on the roles of loading time scale and thermo-mechanical two-field coupling in the deformation domain patterns and stress hysteresis in NiTi SMA. We first report the effects of loading time or stretching rate on the domain patterns and stress hysteresis observed in NiTi strips in the strain rate range of 10-5/s 10-1/s. It was found that the nominal stress-strain curve of the specimen changed from near-isothermal plateau-type with distinct stress-drops in the low strain rate region to the near-adiabatic smooth hardening-type in the high strain rate region. The pseudoelastic hysteresis varies non-monotonically with the external loading rate and the maximum hysteresis occurs at certain intermediate loading rate. The corresponding deformation mode changed from the localized-propagation mode with a few parallelepiped martensite domain patterns to the near-homogeneous multiple nucleation mode with fine alternating austenite-martensite stripes. The analysis shows that the number of the macroscopic domains (domain spacing) of the specimen increased (decreased) with the applied stretching rate in a power-law form, i.e., the higher the loading rate, the finer the deformation pattern. Such observed power-law dependency is originated from the coupling between the material's nonlinear property (formation and growth of domains) and the transfer of heat in the phase transition process. The important roles of heat transfer dynamics and loading time in imposing the emerging length scale in the deformation pattern of the material is demonstrated. For the hysteresis, a simple two-scale thermo-mechanical coupling model is developed to explain the observed rate-dependent hysteresis phenomenon. We incorporate the effects of the released/absorbed heat of the phase transition and the heat transfer on the pseudoelastic hysteresis. An analytical expression for the rate-dependent hysteresis is derived and the theoretical predictions agree quantitatively well with the experiments.

\footnotetext{
1 email: meqpsun@ust.hk
} 